\title{
Associations of Adenovirus Genotypes in Korean Acute Gastroenteritis Patients with Respiratory Symptoms and Intussusception
}

\author{
Jae-Seok Kim, Su Kyung Lee, Dae-Hyun Ko, Jungwon Hyun, Han-Sung Kim, \\ Wonkeun Song, and Hyun Soo Kim
}

Department of Laboratory Medicine, Hallym University College of Medicine, Hwaseong, Republic of Korea

Correspondence should be addressed to Hyun Soo Kim; hskim0901@empas.com

Received 18 September 2016; Revised 16 December 2016; Accepted 27 December 2016; Published 1 February 2017

Academic Editor: Graciela Russomando

Copyright (C) 2017 Jae-Seok Kim et al. This is an open access article distributed under the Creative Commons Attribution License, which permits unrestricted use, distribution, and reproduction in any medium, provided the original work is properly cited.

Human adenoviruses (HAdVs) cause a wide range of diseases, including respiratory infections and gastroenteritis, and have more than 65 genotypes. To investigate the current genotypes of circulating HAdV strains, we performed molecular genotyping of HAdVs in the stool from patients with acute gastroenteritis and tried to determine their associations with clinical symptoms. From June 2014 to May 2016, 3,901 fecal samples were tested for an AdV antigen, and 254 samples (6.5\%) yielded positive results. Genotyping using PCR and sequencing of the capsid hexon gene was performed for $236 \mathrm{AdV}$ antigen-positive fecal specimens. HAdV-41, of species $\mathrm{F}$, was the most prevalent genotype (60.6\%), followed by HAdV-2 of species C (13.8\%). Other genotypes, including HAdV3, HAdV-1, HAdV-5, HAdV-6, HAdV-31, HAdV-40, HAdV-12, and HAdV-55, were also detected. Overall, 119 patients (50.4\%) showed concomitant respiratory symptoms, and 32 patients (13.6\%) were diagnosed with intussusception. HAdV-1 and HAdV-31 were significantly associated with intussusception $(P<0.05)$. Our results demonstrate the recent changes in trends of circulating AdV genotypes associated with gastroenteritis in Korea, which should be of value for improving the diagnosis and developing new detection, treatment, and prevention strategies for broad application in clinical laboratories.

\section{Introduction}

Human adenoviruses (HAdVs) have been associated with a wide range of clinical symptoms, including gastroenteritis, acute respiratory infections, conjunctivitis, hemorrhagic cystitis, and meningoencephalitis $[1,2]$. AdVs are among the main pathogens detected in cases of acute viral infectious diarrhea, especially in children less than 5 years of age [3], and account for 1-31\% of all cases of diarrhea in children [4].

AdVs, belonging to the family Adenoviridae and genus Mastadenovirus, are nonenveloped viruses that are 70$100 \mathrm{~nm}$ in diameter and have linear, double-stranded DNA enclosed by a protein shell (capsid). AdVs are grouped into 6 species $(A-G)$ based on the antigenic variants of the capsid protein and can be further differentiated into 70 HAdV genotypes [5-7]. AdVs have 11 structural proteins, three of which are capsid proteins such as hexons, penton bases, and fibers. In addition, there are group- and type-specific epitopes on both hexons and fibers.

Among the various serotypes of AdVs, two serotypes from species F, AdV-40 and AdV-41, have been clearly associated with infantile diarrhea and are thus referred to as enteric AdVs [2]. Most studies on gastroenteritis caused by AdV have focused on AdV-40 and AdV-41, and most of the commercial AdV detection PCR kits can detect only these two serotypes. However, other types of AdV in stool have been reported in diarrhea patients [2], and recent studies on intussusception have revealed the clinical importance of other serotypes of AdVs [8, 9].

Therefore, we performed molecular genotyping of HAdV strains in stool specimens collected from patients with acute gastroenteritis in Korea from 2014 to 2016, with the aim of investigating the incidence of AdV gastroenteritis, the distribution of $\mathrm{AdV}$ genotypes, the types other than types 40 


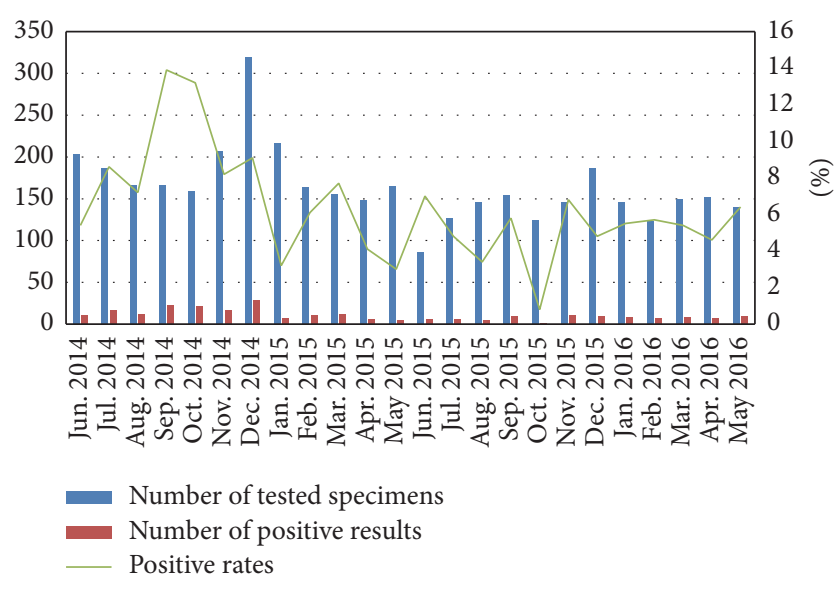

FIgURE 1: Monthly distribution of adenovirus infections in Korean patients with acute gastroenteritis detected by antigen enzymeimmunoassay test.

and 41 that might be associated with gastroenteritis, and the relationship between AdV genotypes and clinical symptoms.

\section{Methods}

2.1. Patient Samples and AdV Antigen Test. From June 2014 to May 2016, 3,901 fecal specimens were tested for the presence of AdV antigen using RIDASCREEN Adenovirus Antigen Test Kit (R-Biopharm, Darmstadt, Germany) in the laboratory of Hallym University Dongtan Sacred Heart Hospital, a 650-bed university hospital in Korea. This kit uses an enzymelinked immunosorbent assay technique for detecting AdV in stool samples and the monoclonal antibodies reactive to the AdV-specific hexon protein and can detect most types of AdV [10]. A total of 254 specimens (6.5\%) showed AdV antigen-positive results. Of these, 236 samples were subjected to AdV PCR and sequencing, and 18 samples could not be further analyzed due to an insufficient stool volume or missing samples. Stool samples were diluted to a $10 \%$ stool suspension in phosphate-buffered saline and stored at $-70^{\circ} \mathrm{C}$ until used for AdV PCR and genotyping. The clinical data collected from the patients' medical records included their age, gender, concomitant respiratory symptoms (cough, sputum, rhinorrhea, pharyngeal injection, and paratonsillar hypertrophy), and intussusception. The age of patients with AdV antigen-positive results ranged from 0 days to 87 years; 220 samples (93.2\%) were from patients less than 5 years old. This study was approved by the Institutional Review Board of Hallym University Dongtan Sacred Heart Hospital (IRB No. 2014-069).

2.2. AdV Genotyping and Sequencing. Viral DNA extraction from fecal suspensions for PCR and genotyping was performed using the QIAamp DNA mini Kit (Qiagen, Hilden, Germany) and the QIAcube platform (Qiagen). AdV hexon genotyping was performed by PCR and sequencing using a specified primer set (ADHEX1F/AD2) according to previous studies with few modifications $[11,12]$. For DNA extracts that could not be amplified by this primer set, a different primer set (AD1/AD2) was used for PCR [12]. The PCR products were visualized by electrophoresis on an agarose gel and analyzed by DNA sequencing. The nucleotide sequences were analyzed using ABI Prism BigDye Terminator version 3.1 (Applied Biosystems, Foster City, CA, USA), and genotypes were confirmed using the NCBI BLAST server of the GenBank database.

2.3. Statistical Analysis. Positive rates of each genotype were compared to those of the total group or other groups using Fisher's exact test or chi-square test. Titers of AdV antigen (estimated from the optical density [OD]) of each genotype were compared to those of the total group using the Student $t$-test. The tests were considered statistically significant for $P$ values $<0.05$. MedCalc version 15 (MedCalc Software, Mariakerke, Belgium) was used for all statistical analyses.

\section{Results}

3.1. Monthly Distribution of AdV-Positive Cases. From June 2014 to May 2016, the highest positive rates among results over $10 \%$ from the AdV antigen test were observed in September and October of 2014 (13.9\% and 13.2\%), and the lowest positive rate was observed in October of 2015 $(0.8 \%)$; the average positive rate was $6.5 \%$. There was no seasonal peak detected, as positive AdV cases were observed throughout the study period (Figure 1).

3.2. AdV Genotype and Clinical Manifestations. The distribution of HAdV genotypes and their associations with clinical characteristics are summarized in Table 1. Of the 236 genotyped specimens, HAdV-41 was the most prevalent genotype, followed by HAdV-2. Other genotypes, including HAdV3, HAdV-1, HAdV-5, HAdV-6, HAdV-31, AdV-40, AdV-12, and HAdV-55, were also detected. A total of 119 patients (50.4\%) showed concomitant respiratory symptoms, and those infected with HAdV-2 (species C) showed significantly increased frequencies of respiratory symptoms $(P<0.01)$. Thirty-two patients (13.6\%) were diagnosed with intussusception, including 9 of 14 (64.3\%) patients infected with HAdV1 (species C) but only 4 of 132 (3.0\%) patients infected with HAdV-41 (species F). The rate of intussusception of other species $\mathrm{C}$ genotypes ranged from $26.7 \%$ to $50.0 \%$, and the overall rate among patients infected with species $\mathrm{C} A d V$ s was $37.9 \%$. In addition, 3 of 4 patients infected with HAdV-31 (species A) had intussusception.

3.3. AdV Viral Load according to Genotype. The average $\mathrm{OD}$ value of the enzyme-linked immunosorbent assay for detection of the AdV antigen (reflecting the viral load) was highest in the HAdV-41 and HAdV-40 groups, while the lowest viral loads were observed in the HAdV-3 and HAdV55 groups (Table 1). The average OD value of AdV antigen was significantly increased in the HAdV-41 group and was significantly decreased in the HAdV-2 and HAdV-3 groups $(P<0.05)$ compared to the value for the total group.

3.4. Distribution of AdV Genotypes according to Age Group. Table 2 shows the age distribution of patients with genotyped 


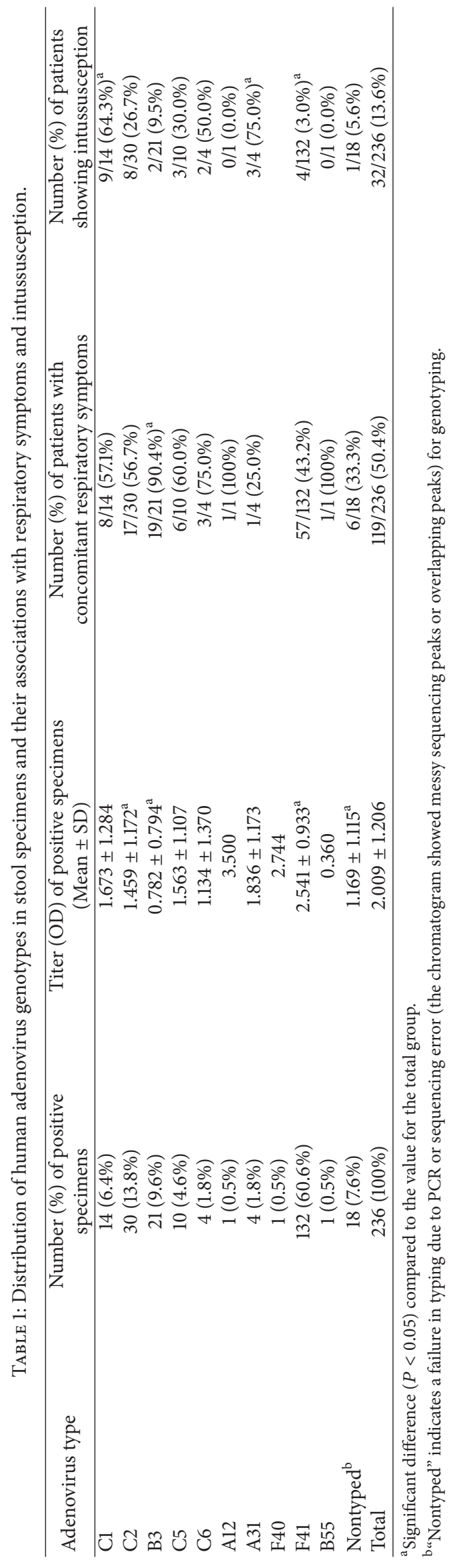


TABLE 2: Distribution of human adenovirus genotypes according to patient age.

\begin{tabular}{|c|c|c|c|c|c|c|c|c|}
\hline Genotype & $0-1 \mathrm{yr}$ & $1-2 \mathrm{yr}$ & $2-3 \mathrm{yr}$ & $3-4 \mathrm{yr}$ & $4-5 \mathrm{yr}$ & $\geq 5 \mathrm{yr}$ & Total & $P$ value \\
\hline $\mathrm{C} 1$ & 7 & 3 & 3 & 1 & & & 14 & $\mathrm{~ns}^{\mathrm{a}}$ \\
\hline $\mathrm{C} 2$ & 14 & 10 & 2 & 3 & 1 & & 30 & ns \\
\hline B3 & 4 & 5 & 3 & 3 & 3 & 3 & 21 & ns \\
\hline C5 & 7 & 3 & & & & & 10 & ns \\
\hline C6 & 3 & 1 & & & & & 4 & ns \\
\hline A12 & 1 & & & & & & 1 & ns \\
\hline A31 & 3 & 1 & & & & & 4 & ns \\
\hline $\mathrm{F} 40$ & 1 & & & & & & 1 & ns \\
\hline F41 & 43 & 46 & 21 & 11 & 7 & 4 & 132 & ns \\
\hline B55 & & & 1 & & & & 1 & ns \\
\hline Nontyped & 3 & 3 & 2 & & 1 & 9 & 18 & ns \\
\hline Total & 86 & 72 & 32 & 18 & 12 & 16 & 236 & \\
\hline
\end{tabular}

${ }^{a}$ ns $=$ not statistically significant.

TABLE 3: Comparison of results with previously reported adenovirus genotypes.

\begin{tabular}{|c|c|c|c|c|c|c|c|}
\hline Country & Korea & Korea & China & China & Japan & Japan & Tanzania \\
\hline Year of specimen collection & 2014-2016 & 2004-2006 & 2011-2012 & 2010-2011 & 1995-2009 & 2009-2014 & 2010-2011 \\
\hline Number of typed specimens & 236 & 113 & 219 & 31 & Not presented & Not presented & 37 \\
\hline AdV-C1 & $14(6.4 \%)$ & $0(0.0 \%)^{\mathrm{a}}$ & Detected & & Detected & Detected & $3(8.1 \%)$ \\
\hline $\mathrm{AdV}-\mathrm{C} 2$ & $30(13.8 \%)$ & $2(1.7 \%)^{\mathrm{a}}$ & $\sim 13 \%$ & $1(3.2 \%)$ & Detected & Detected & $3(8.1 \%)$ \\
\hline AdV-B3 & $21(9.6 \%)$ & $11(9.7 \%)$ & Detected & $7(22.6 \%)$ & Detected & Detected & $1(2.7 \%)$ \\
\hline AdV-E4 & & $1(0.8 \%)$ & Detected & & & Detected & \\
\hline AdV-C5 & $10(4.6 \%)$ & & & $1(3.2 \%)$ & Detected & Detected & \\
\hline AdV-C6 & $4(1.8 \%)$ & & Detected & & & & \\
\hline AdV-B7 & $0(0.0 \%)$ & $5(4.4 \%)^{\mathrm{a}}$ & $12-15 \%$ & & & & $3(8.1 \%)$ \\
\hline AdV-B11 & & & Detected & & & & \\
\hline AdV-A12 & $1(0.5 \%)$ & & Detected & $2(6.5 \%)$ & & & \\
\hline AdV-B14 & & & Detected & & & & \\
\hline AdV-A18 & & & & & & & $3(8.1 \%)$ \\
\hline AdV-D19 & & & & & & & $1(2.7 \%)$ \\
\hline AdV-A31 & $4(1.8 \%)$ & $2(1.7 \%)$ & $16-25 \%$ & & & Detected & $5(13.5 \%)$ \\
\hline AdV-D37 & & $1(0.8 \%)$ & & & & & \\
\hline AdV-F40 & $1(0.5 \%)$ & $3(2.6 \%)$ & $\sim 11 \%$ & $5(16.1 \%)$ & Detected & & $10(27.0 \%)$ \\
\hline AdV-F41 & $132(60.6 \%)$ & $54(47.8 \%)$ & $38-46 \%$ & $14(45.2 \%)$ & Detected & $71.3 \%$ & $8(21.6 \%)$ \\
\hline AdV-B55 & $1(0.5 \%)$ & & & & & & \\
\hline Nontyped & $18(7.6 \%)$ & & & & & & \\
\hline References & This study & {$[13]$} & [14] & [15] & [16] & [17] & [3] \\
\hline
\end{tabular}

${ }^{\text {a Significant difference }(}(P<0.05)$ compared to the frequency of the same adenovirus type observed in this study.

AdVs. The frequencies of other types were not significantly different among different age groups.

\section{Discussion}

In this study, we genotyped AdVs identified in clinical stool samples from acute gastroenteritis patients in Korea. Few studies have examined AdV genotypes other than the most common types 40/41 in stool samples. In this study, HAdV-41 was the most prevalent genotype, which is an enteric $\mathrm{AdV}$, although we also detected several other genotypes in our patients. These findings are similar to those previously reported [3, 13-17], with some interesting differences (Table 3). First, the $\mathrm{C} 1$ genotype was newly detected in Korean patients. Second, the prevalence of the C2 genotype was significantly increased compared to detection rates previously reported in a similar population during 2004-2006. Third, the B7 genotype was not detected in the present study. Finally, this is the first report of AdV B55 detection in stool samples, which is commonly known as a genotype associated with respiratory infection [18].

There are $70 \mathrm{AdV}$ genotypes showing high genetic diversity, which has hindered the design of a single primer set for the detection of all genotypes. The two primers sets 
used in this study were proven to be suitable for detecting AdV-1, AdV-2, AdV-3, AdV-5, AdV-6, AdV-12, AdV-31, AdV40, AdV-41, and AdV-55 in stool samples. We performed in silico analysis to confirm whether our primer sets could detect at least one of each HAdV type from HAdV-1 to HAdV-69, which showed less than four base-mismatches between our primer sequences and the $69 \mathrm{AdV}$ types (data not shown). However, it was not clear whether this analysis predicted the successful amplification of the different strains, and the method used may fail at detecting variants with mismatches in primer-binding sites, despite the in silico primer set affinity results. Moreover, considering the high genetic diversity among AdVs, strains with single nucleotide variations at primer-annealing sites cannot be detected using these two primer sets. The majority of previous studies on the role of $\mathrm{AdV}$ in gastroenteritis have focused on the two main enteric genotypes (HAdV-40 and HAdV-41), and our study suggests that these detection methods are not suitable for identifying other genotypes. Although enteric AdVs are the most common cause of gastroenteritis, other genotypes can also be associated with enteric symptoms. Our results highlight the need to develop a more efficient set of primers for the detection of a wider range of genotypes, rather than restricting analyses to the most common types associated with certain symptoms.

In this study, the overall positive rate of the AdV antigen test in stool was $6.6 \%$, and we did not observe a seasonal peak in the distribution of AdV gastrointestinal infections. Nevertheless, seasonal variation of gastroenteric illness linked to $\mathrm{AdV}$ infection remains a controversial topic, as peak incidences were reported in Korea ( $>10 \%)$ from August to September of 2004-2006 and in China in May and October of 2010 [13, 19]. However, no seasonal variation was detected in another study conducted in China during 2011-2012 [14].

To the best of our knowledge, no study has evaluated the association between the AdV antigen concentration in stool samples, the genotype detected, and concomitant respiratory symptoms. Patients infected with HAdV-40 and HAdV-41 had higher AdV antigen titers and a lower frequency of concomitant respiratory symptoms, which suggests that these types of AdV are primarily associated with gastroenteritis. HAdV-3 had a lower AdV titer and was significantly associated with respiratory symptoms, which suggests that AdV3 is primarily associated with respiratory infections, and can be secondarily secreted into the stool. However, it is technically impossible to demonstrate a causative role for any AdV type other than those known to be enteric. It is possible that AdV types infecting the respiratory tract will be shed in the feces over prolonged periods of time [20,21]. This applies to HAdV-C1, HAdV-B3, HAdV-C5, HAdV-C6, and HAdV-B55, especially if the patient has an active or has had a recent respiratory infection associated with one of these types. HAdV-A12 and HAdV-A31 have been reported to be associated with gastroenteritis, and therefore it is not surprising that their viral loads in stool samples were high (Table 2). Moreover, asymptomatic viral shedding in the stool from healthy children has been commonly reported [22, 23]. Therefore, caution should be taken when diagnosing adenoviral gastroenteritis based on the detection of AdV in stool.
In this study, 32 patients (13.6\%) were diagnosed with intussusception, and the highest rate was observed in those infected with species C (37.9\%). Other studies have described an association between $\mathrm{AdV}$ and intussusception [8, 9, 24]. In Australia during 2008-2011, HAdV species C was detected more frequently in cases than controls with $31 / 74$ (41.9\%) of cases testing positive compared to $39 / 289$ (13.49\%) controls [9]. In Thailand, all of the HAdVs detected $(n=12)$ in intussusception patients $(n=40)$ were reported to be of species $\mathrm{C}$, and among the 44 intussusception patients identified in a study in Korea 22 (78.6\%) had nonenteric AdVs, and AdV species C comprised the majority, with 20 cases (90.9\%) [24].

\section{Conclusions}

In summary, HAdV-41 was the most frequent genotype isolated from patients with acute gastroenteritis in Korea in 2014-2016, and we found that other types of AdVs, which are known to be associated with respiratory infections, were detected in patients with acute gastroenteritis. We have expanded the list of AdV genotypes detected in stool samples and their association with respiratory symptoms and intussusception. Our results demonstrate the recent changes in trends of circulating $\mathrm{AdV}$ genotypes linked to acute gastroenteritis in Korea, which should be of value for improving the diagnosis and developing new detection, treatment, and prevention strategies for broad application in clinical laboratories.

\section{Competing Interests}

The authors declare that there is no conflict of interests regarding the publication of this paper.

\section{Acknowledgments}

This study was supported by the Technology Innovation Program (10047748) funded by the Ministry of Trade, Industry \& Energy and by Hallym University Research Fund 2016 (HURF-2016-24). The authors appreciate Ji Sun Noh for the excellent technical assistance.

\section{References}

[1] R.-F. Chen and C.-Y. Lee, "Adenoviruses types, cell receptors and local innate cytokines in adenovirus infection," International Reviews of Immunology, vol. 33, no. 1, pp. 45-53, 2014.

[2] O. Ruuskanen, J. P. Metcalf, O. Meurman, and G. Akusjärvi, "Adenoviruses," in Clinical Virology, pp. 559-579, American Society of Microbiology, 3rd edition, 2009.

[3] S. J. Moyo, K. Hanevik, B. Blomberg et al., "Prevalence and molecular characterisation of human adenovirus in diarrhoeic children in Tanzania; A Case Control Study," BMC Infectious Diseases, vol. 14, no. 1, article 666, 2014.

[4] I. Wilhelmi, E. Roman, and A. Sánchez-Fauquier, "Viruses causing gastroenteritis," Clinical Microbiology and Infection, vol. 9, no. 4, pp. 247-262, 2003.

[5] Y. Chen, F. Liu, C. Wang et al., "Molecular identification and epidemiological features of human adenoviruses associated with acute respiratory infections in hospitalized children in 
Southern China, 2012-2013," PLoS ONE, vol. 11, no. 5, Article ID e0155412, 2016.

[6] G. Singh, C. M. Robinson, S. Dehghan et al., "Homologous recombination in E3 genes of human adenovirus species D," Journal of Virology, vol. 87, no. 22, pp. 12481-12488, 2013.

[7] E. Hage, U. Gerd Liebert, S. Bergs, T. Ganzenmueller, and A. Heim, "Human mastadenovirus type 70: a novel, multiple recombinant species D mastadenovirus isolated from diarrhoeal faeces of a haematopoietic stem cell transplantation recipient," The Journal of General Virology, vol. 96, no. 9, pp. 2734-2742, 2015.

[8] N. Ukarapol, P. Khamrin, J. Khorana, J. Singhavejsakul, A. Damrongmanee, and N. Maneekarn, "Adenovirus infection: a potential risk for developing intussusception in pediatric patients," Journal of Medical Virology, vol. 88, no. 11, pp. 1930-1935, 2016.

[9] C. A. Minney-Smith, A. Levy, M. Hodge et al., "Intussusception is associated with the detection of adenovirus $\mathrm{C}$, enterovirus $\mathrm{B}$ and rotavirus in a rotavirus vaccinated population," Journal of Clinical Virology, vol. 61, no. 4, pp. 579-584, 2014.

[10] J. Kim, H. S. Kim, H.-S. Kim et al., "Evaluation of an immunochromatographic assay for the rapid and simultaneous detection of rotavirus and adenovirus in stool samples," Annals of Laboratory Medicine, vol. 34, no. 3, pp. 216-222, 2014.

[11] I. Casas, A. Avellon, M. Mosquera et al., "Molecular identification of adenoviruses in clinical samples by analyzing a partial hexon genomic region," Journal of Clinical Microbiology, vol. 43, no. 12, pp. 6176-6182, 2005.

[12] W. Xu, M. C. McDonough, and D. D. Erdman, "Species-specific identification of human adenoviruses by a multiplex PCR assay," Journal of Clinical Microbiology, vol. 38, pp. 4114-4120, 2000.

[13] J. I. Lee, G.-C. Lee, J. Y. Chung et al., "Detection and molecular characterization of adenoviruses in Korean children hospitalized with acute gastroenteritis," Microbiology and Immunology, vol. 56, no. 8, pp. 523-528, 2012.

[14] L. Liu, Y. Qian, Y. Zhang, J. Deng, L. Jia, and H. Dong, "Adenoviruses associated with acute diarrhea in children in Beijing, China," PLoS ONE, vol. 9, no. 2, Article ID e88791, 2014.

[15] L. Lu, R. Jia, H. Zhong et al., "Molecular characterization and multiple infections of rotavirus, norovirus, sapovirus, astrovirus and adenovirus in outpatients with sporadic gastroenteritis in Shanghai, China, 2010-2011," Archives of Virology, vol. 160, no. 5, pp. 1229-1238, 2015.

[16] S. K. Dey, I. Hoq, S. Okitsu, S. Hayakawa, and H. Ushijima, "Prevalence, seasonality, and peak age of infection of enteric adenoviruses in Japan, 1995-2009," Epidemiology and Infection, vol. 141, no. 5, pp. 958-960, 2013.

[17] A. Thongprachum, P. Khamrin, N. Maneekarn, S. Hayakawa, and H. Ushijima, "Epidemiology of gastroenteritis viruses in Japan: prevalence, seasonality, and outbreak," Journal of Medical Virology, vol. 88, no. 4, pp. 551-570, 2016.

[18] B. Cao, G.-H. Huang, Z.-H. Pu et al., "Emergence of community-acquired adenovirus type 55 as a cause of communityonset pneumonia," Chest, vol. 145, no. 1, pp. 79-86, 2014.

[19] L.-Y. Liu, Y. Qian, Y. Zhang, L.-P. Jia, H.-J. Dong, and J. Deng, "Investigation of adenovirus infection in hospitalized children with diarrhea during 2010 in Beijing, China," Chinese Journal of Pediatrics, vol. 50, no. 6, pp. 450-454, 2012.

[20] A. Lyons, J. Longfield, R. Kuschner et al., "A double-blind, placebo-controlled study of the safety and immunogenicity of live, oral type 4 and type 7 adenovirus vaccines in adults," Vaccine, vol. 26, no. 23, pp. 2890-2898, 2008.
[21] S. Ye, D. M. Whiley, R. S. Ware et al., "Detection of viruses in weekly stool specimens collected during the first 2 years of life: a pilot study of five healthy Australian infants in the rotavirus vaccine era," Journal of Medical Virology, 2016.

[22] B. Kapusinszky, P. Minor, and E. Delwart, "Nearly constant shedding of diverse enteric viruses by two healthy infants," Journal of Clinical Microbiology, vol. 50, no. 11, pp. 3427-3434, 2012.

[23] O. Cinek, E. Witsø, S. Jeansson et al., "Longitudinal observation of enterovirus and adenovirus in stool samples from Norwegian infants with the highest genetic risk of type 1 diabetes," Journal of Clinical Virology, vol. 35, no. 1, pp. 33-40, 2006.

[24] Y. W. Lee, S. I. Yang, J. M. Kim, and J. Y. Kim, "Clinical features and role of viral isolates from stool samples of intussuception in children," Pediatric Gastroenterology, Hepatology and Nutrition, vol. 16, no. 3, pp. 162-170, 2013. 

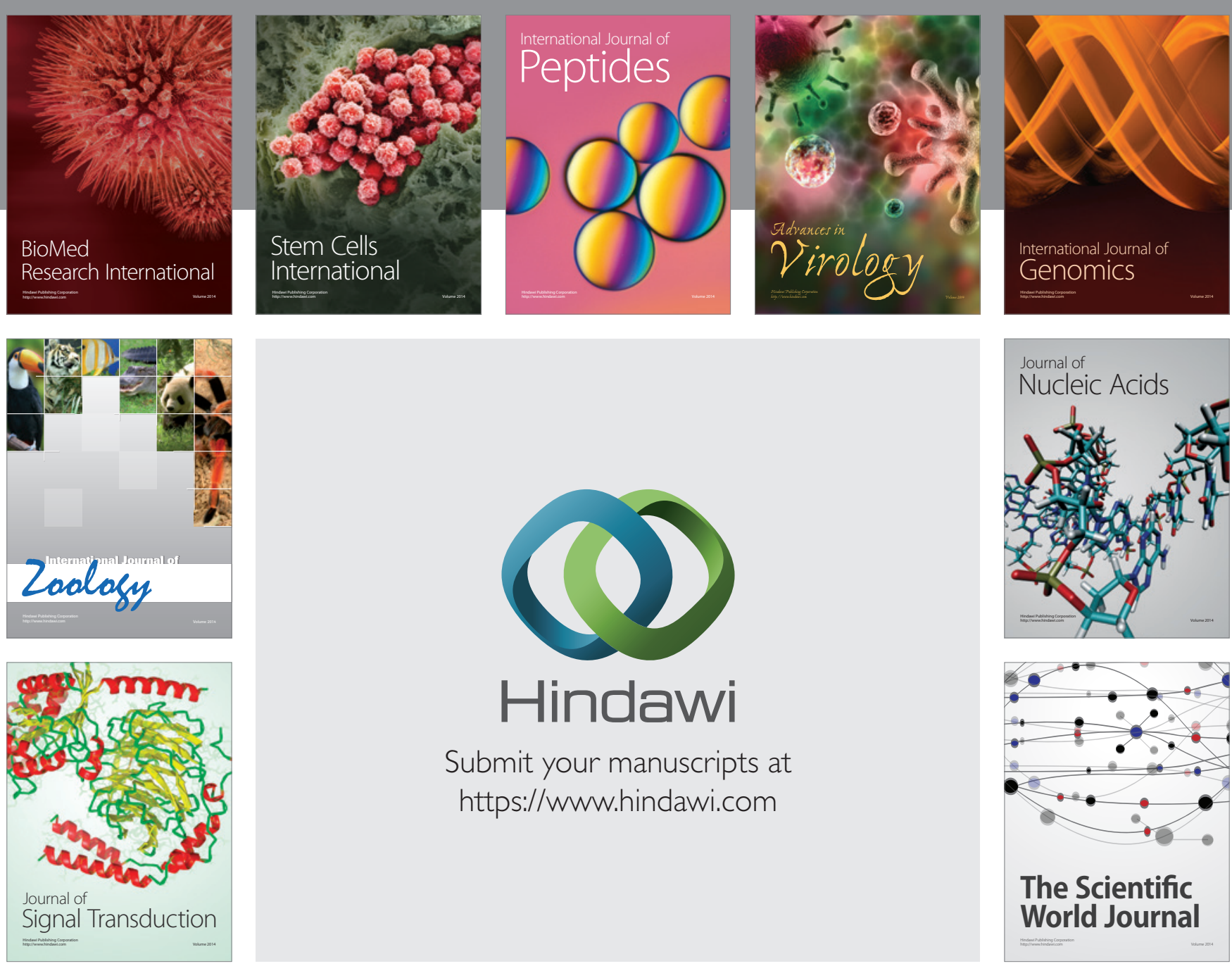

Submit your manuscripts at

https://www.hindawi.com
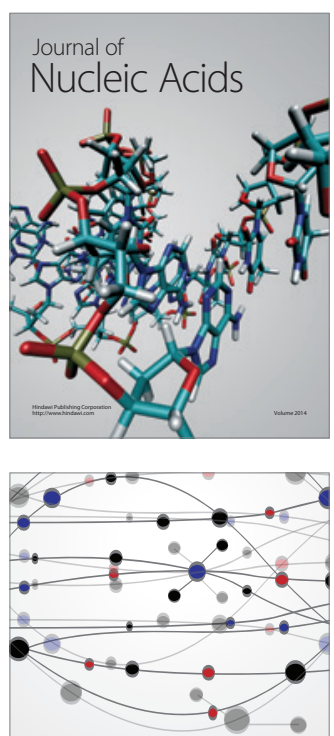

The Scientific World Journal
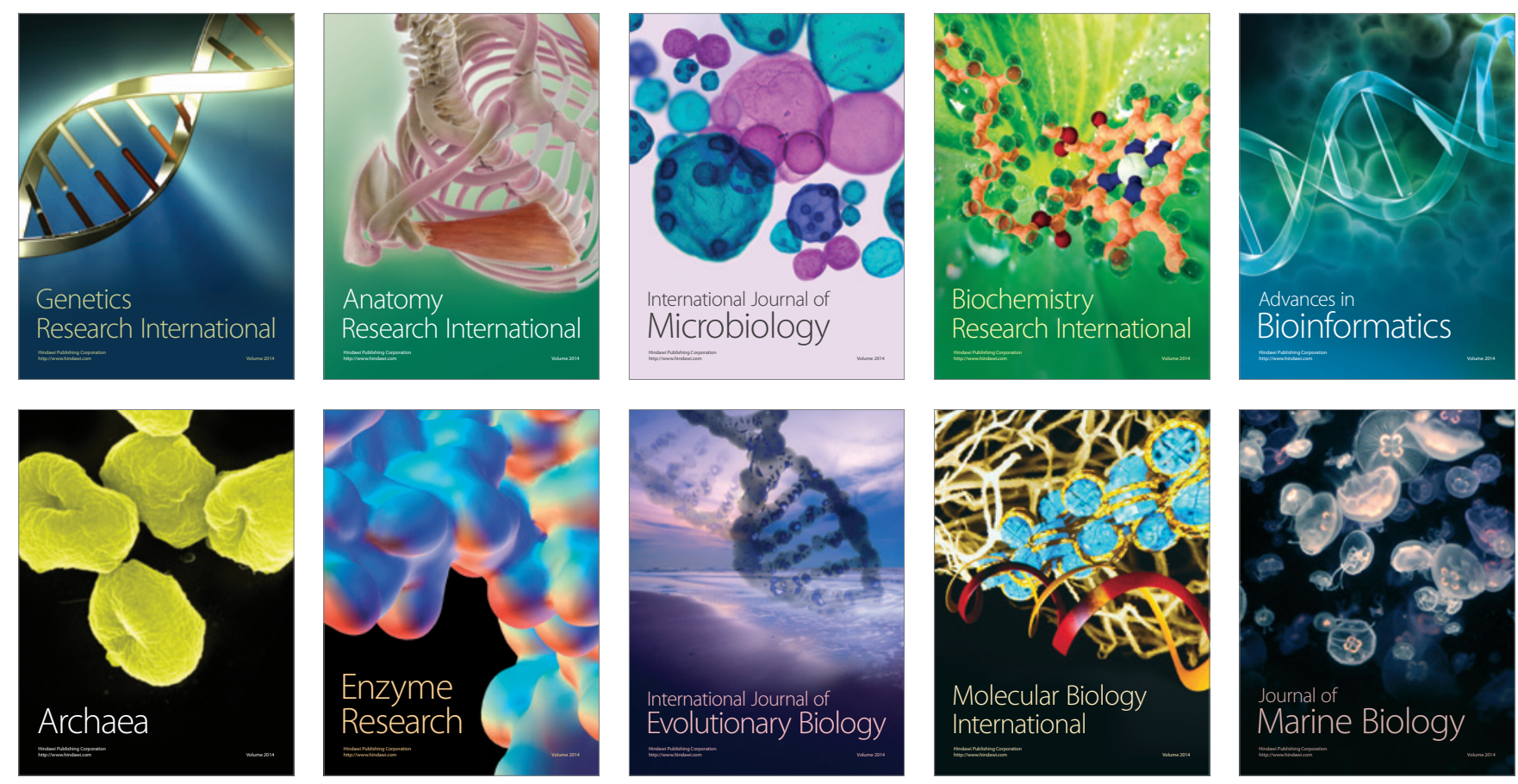